\title{
Anti-mesothelin vaccine CRS-207 with or without low-dose cyclophosphamide plus chemotherapy as front-line treatment for malignant pleural mesothelioma (MPM)
}

\author{
Raffit Hassan ${ }^{1 *}$, Evan Alley ${ }^{2}$, Hedy Kindler ${ }^{3}$, Scott Antonia ${ }^{4}$, Thierry Jahan ${ }^{5}$, Anish Thomas ${ }^{1}$, Somayeh Honarmand ${ }^{6}$, \\ Aimee L Murphy ${ }^{6}$, John J Grous ${ }^{7}$, Dirk G Brockstedt ${ }^{6}$
}

From 30th Annual Meeting and Associated Programs of the Society for Immunotherapy of Cancer (SITC 2015) National Harbor, MD, USA. 4-8 November 2015

\section{Background}

CRS-207 is live-attenuated, double-deleted Listeria monocytogenes (LADD) engineered to express the tumor-associated antigen mesothelin which is highly expressed in malignant pleural mesothelioma (MPM). CRS-207 stimulates potent innate and adaptive immunity and in combination with chemotherapy may act synergistically to alter the tumor environment to be more susceptible to immune-mediated killing. Preliminary data of 32 patients who received CRS-207 in combination with pemetrexed/cisplatin showed $60 \%$ partial responses and 94\% disease control[1]. Low-dose cyclophosphamide (Cy) in combination with LADD improved immune and anti-tumor responses and overall survival in preclinical studies.

\section{Methods}

Up to 60 subjects are planned to be enrolled in 2 mutually exclusive, sequential cohorts at 5 clinical trial sites. Patients must be chemotherapy-naïve, have unresectable MPM, good performance status (ECOG 0 or 1 ) and adequate organ function. Eligible patients in Cohort 1 receive 2 prime vaccinations with CRS-207 $\left(1 \times 10^{9}\right.$ CFU; $250 \mathrm{~mL}$ IV over 2 hours) 2 weeks apart, followed by up to 6 cycles of pemetrexed $\left(500 \mathrm{mg} / \mathrm{m}^{2}\right)$ and cisplatin $\left(75 \mathrm{mg} / \mathrm{m}^{2}\right)$ 3 weeks apart and 2 CRS-207 boost vaccinations 3 weeks apart. Subjects are followed every 8 weeks until disease progression. Clinically stable patients continue CRS-207 maintenance vaccinations every 8 weeks. Patients in Cohort 2 receive low-dose Cy $\left(200 \mathrm{mg} / \mathrm{m}^{2}\right) 1$ day prior to each CRS-207 vaccination. Objectives of the study are safety, immunogenicity, objective tumor responses and tumor marker kinetics.

\section{Authors' details}

${ }^{1}$ Center for Cancer Research at the National Institutes of Health, Bethesda, MD, USA. ${ }^{2}$ University of Pennsylvania, Philadelphia, PA, USA. ${ }^{3}$ The University of Chicago Medicine, Chicago, IL, USA. ${ }^{4}$ H. Lee Moffitt Cancer Center and Research Institute, Tampa, FL, USA. ${ }^{5}$ University of California San Francisco, San Francisco, CA, USA. ${ }^{6}$ Aduro Biotech Inc., Berkeley, CA, USA. ${ }^{7}$ Aduro Biotech Inc., Hopedale, MA, USA.

Published: 4 November 2015

\section{Reference}

1. Hassan, Antonia, Alley, et al: Mesothelin-targeted immunotherapy CRS207 in combination with standard of care chemotherapy as treatment for malignant pleural mesothelioma (MPM). J Clin Oncol 2015, 33(suppl) abstr 7565 .

doi:10.1186/2051-1426-3-S2-P161

Cite this article as: Hassan et al:: Anti-mesothelin vaccine CRS-207 with or without low-dose cyclophosphamide plus chemotherapy as frontline treatment for malignant pleural mesothelioma (MPM). Journal for ImmunoTherapy of Cancer 2015 3(Suppl 2):P161. 